\title{
Nickel-Catalyzed Benzylic Alkynylation: Migratory Hydroalkynylation and Enantioselective Hydroalkynylation of Olefins with Bromoalkynes
}

\author{
Xiaoli Jiang, Bo Han, Yuhang Xue, Mei Duan, Zhuofan Gui, You Wang, ${ }^{*}$ and Shaolin Zhu*
}
[*] X. Jiang, B. Han, Y. Xue, M. Duan, Z. Gui, Dr. Y. Wang, Prof. S. Zhu
State Key Laboratory of Coordination Chemistry, Jiangsu Key Laboratory of Advanced Organic Materials, Chemistry and Biomedicine Innovation Center (ChemBIC), School of Chemistry and Chemical Engineering, Nanjing University, Nanjing, 210093, (China)
E-mail: shaolinzhu@nju.edu.cn; wangyou@nju.edu.cn
Supporting information for this article is given via a link at the end of the document.

\begin{abstract}
A nickel-hydride catalyzed reductive migratory hydroalkynylation of olefins with bromoalkynes that delivers the corresponding benzylic alkynylation products in high yield and with excellent regioselectivity has been developed. Catalytic enantioselective hydroalkynylation of styrenes has been realized using a simple chiral PyrOx ligand. The obtained enantioenriched benzylic alkynes are versatile synthetic intermediates and can be readily transformed into synthetically useful chiral synthons.
\end{abstract}

Owing to its low-cost, facile oxidative addition, and availability of diverse oxidation states, nickel has emerged as a catalyst complementary to palladium over the past two decades, especially in cross-coupling reaction involving $\mathrm{C}\left(\mathrm{sp}^{3}\right)$ fragments. ${ }^{[1]}$ Reductive migratory hydrofunctionalization catalyzed by nickel hydride ${ }^{[2,3]}$ has recently been recognized as an alternative protocol for selective functionalization of remote $\mathrm{C}\left(\mathrm{sp}^{3}\right)-\mathrm{H}$ bonds (Figure 1a). ${ }^{[4-13]}$ Compared to conventional cross-coupling, this process (i) employs readily available, benchstable alkenes or alkene precursors instead of specially generated organometallic reagents as starting materials and (ii) could selectively functionalize a remote $\mathrm{C}\left(\mathrm{sp}^{3}\right)-\mathrm{H}$ site instead of the conventional ipso-position. Since its conception, significant progress has been made toward this synthetically useful process ${ }^{[8-11]}$ which requires that the cross-coupling partner (e.g., aryl halide or alkyl halide) could selectively capture an alkylnickel species generated through iterative migratory insertion/ $\beta$-hydride elimination.

To explore this nickel-catalyzed migratory hydrofunctionalization further, we recently investigated if a bromoalkyne, an unsaturated $\mathrm{C}(\mathrm{sp})$ cross-coupling partner which is potentially reactive towards $\mathrm{NiH}$, could be used to achieve remote hydroalkynylation (Figure $1 \mathrm{~b}, \mathrm{i}$ ). Successful implementation of this transformation will require (i) a hydrometalation process that can discriminate between alkene and alkyne and (ii) an alkynylation process highly selective for one of the alkylnickel species. A chiral alkyne bearing an a-arylsubstituted stereogenic $\mathrm{C}\left(\mathrm{sp}^{3}\right)$ center ${ }^{[14]}$ would be ultimately obtained from styrene in such an enantioselective reaction (Figure 1b, ii). Such a transformation would provide direct access to a variety of chiral alkynes, a key structural element in synthesis of bioactive molecules, and other functional materials
(Figure 1c). ${ }^{[15]}$ Here we report the successful execution of this reaction. 
a Remote hydrofunctionalization through reductive $\mathrm{NiH}$ catalysis i conventional cross-coupling

$$
\begin{aligned}
& \overbrace{\text { pregenerated }}^{\mathrm{M}}+\mathrm{FG}-\mathrm{X}-\mathrm{Ni} \rightarrow \\
& \text { organometallic reagent partners cross coupling }
\end{aligned}
$$

ii $\mathrm{NiH}$-catalyzed reductive remote hydrofunctionalization

$$
\begin{gathered}
\text { cross-coupling } \\
\text { partners } \\
\text { (unrefined alkene) }
\end{gathered}
$$

Highlights: avoid organometallic reagents both remote \& ipso coupling

b This work: chemo- \& stereoselective $\mathrm{NiH}$-catalyzed benzylic alkynylation i $\mathrm{NiH}$-catalyzed migratory hydroalkynylation

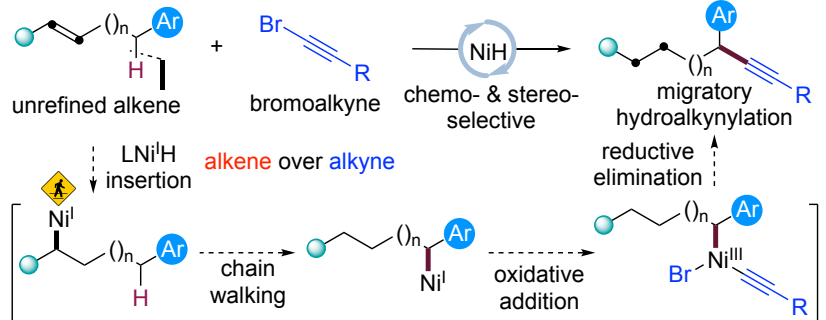

- chemo- \& regioselective $\mathbf{w}$ wo organometallic reagents $\mathbf{m i l d} \&$ broad scope ii Enantioselective $\mathrm{NiH}$-catalyzed hydroalkynylation of vinylarenes

$$
\underset{\text { styrene }}{\mathrm{Br}}=\mathrm{R} \underset{\text { bromoalkyne }}{-\mathrm{NiH}} \rightarrow
$$

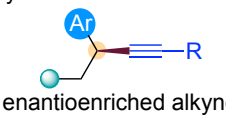

$\mathrm{L}^{*} \mathrm{Ni}{ }^{\prime} \mathrm{H}$ syn-hydrometallation

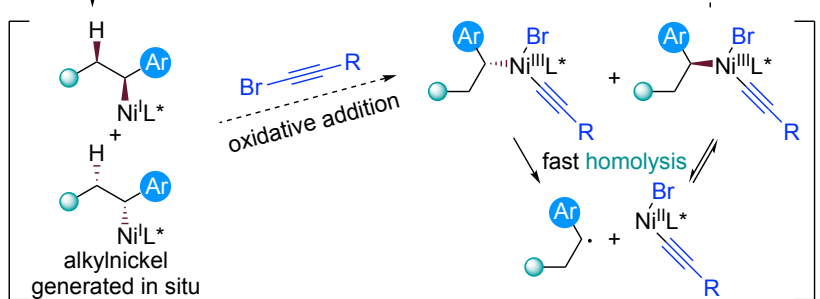

c Representative chiral alkynes in natural products and pharmaceuticals<smiles>C#C[C@@H]1CC[C@]2(C)C=CC(=O)C[C@H]2C1</smiles>

(-)-Chamaecynone (a GPR40 agonist for type 2 diabetes) (anti HIV) versatile synthons $\mathbf{a}$ chemical probe $\mathbf{a}$ bioactive molecules (natural product)

Figure 1. Nickel(I)-hydride catalyzed migratory hydroalkynylation and enantioselective hydroalkynylation.

Our initial studies involved the migratory hydroalkynylation of 4-phenyl-1-butene (1a) using 1-bromo-2(triisopropylsilyl)acetylene (2a) as an alkynylation reagent. It was determined that $\mathrm{Nil}_{2} \cdot \mathrm{xH}_{2} \mathrm{O}$ and the bathocuproine ligand (L) could generate the desired migratory alkynylation product as a single regioisomer [rr (benzylic product: all other isomers) > 99:1] in $82 \%$ yield (entry 1). Other nickel sources such as $\mathrm{NiBr}_{2}$ led to lower yields and a moderate $\mathrm{rr}$ (entry 2). Ligand screening revealed that the previously used ligand, ${ }^{\left[{ }^{[b]}\right]} 6,6^{\prime}$-dimethyl-2,2'bipyridine (L1) resulted in significantly lower yield and $\mathrm{rr}$ (entry 3 ) while a similar ligand neocuproine (L2) led to a similar regioselectivity but a lower yield (entry 4 ). Other silanes such as trimethoxysilane and diethoxymethylsilane gave diminished yields (entries 5 and 6 ) and $\mathrm{K}_{3} \mathrm{PO}_{4} \cdot \mathrm{H}_{2} \mathrm{O}$ was shown to be an unsuitable base (entry 7). Addition of $\mathrm{Nal}$ as an additive improves both the yield and $\mathrm{rr}$ (entry 8). An evaluation of solvents revealed that THF was less effective than DME (entry 9) and conducting the reaction at $40{ }^{\circ} \mathrm{C}$ gave inferior results (entry 10).

Table 1: Variation of reaction parameters.

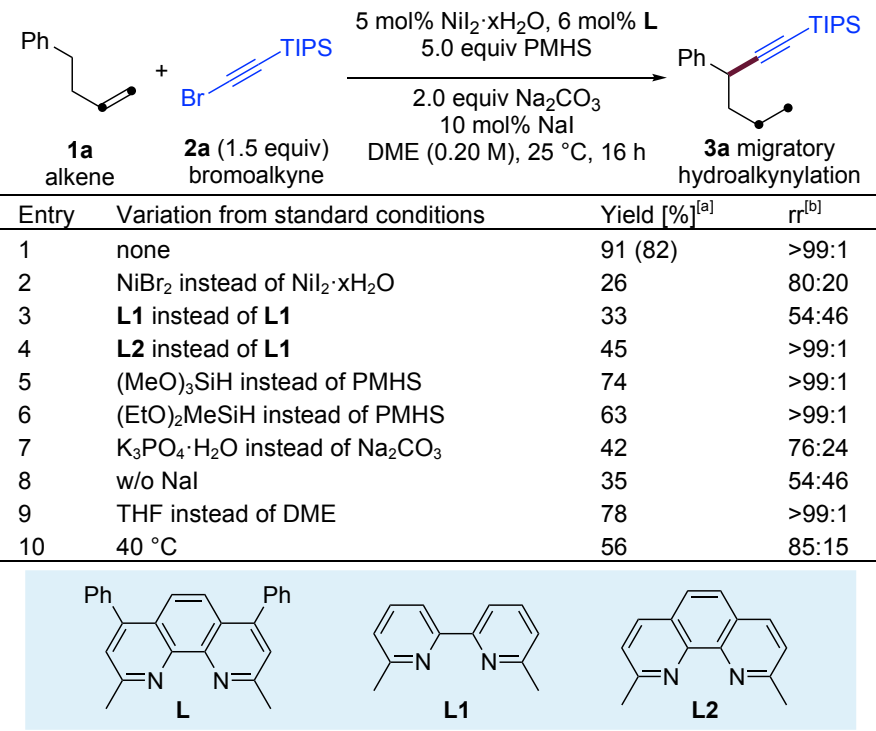

[a] Yields determined by GC using $n$-dodecane as the internal standard, the yield in parentheses is the isolated yield. [b] rr refers to regioisomeric ratio, representing the ratio of the major product to the sum of all other isomers as determined by GC analysis. PMHS = polymethylhydrosiloxane; DME = dimethoxyethane; TIPS = triisopropylsilyl.

With these optimal reaction conditions, we examined the generality of the reaction. As shown in Table 2a, unactivated terminal alkenes bearing electron donating $(\mathbf{3 c})$ or electron withdrawing $(\mathbf{3} \mathbf{d}-\mathbf{3 g})$ substituents on the remote aryl ring are tolerated. A variety of functional groups are readily accommodated, including ethers $(\mathbf{3 c}, \mathbf{3 h}-\mathbf{3 I})$, a trifluoromethyl group (3d), and esters $(\mathbf{3 g}, \mathbf{3 i})$. Importantly, tosylates $(\mathbf{3 j})$ and triflate (3k) commonly used for further cross-coupling, all remained intact. Remarkably, both silyl and sterically hindered alkyl substituted ethynyl bromides work well in this reaction (3I, $3 \mathrm{~m})$. Moreover, a variety of unactivated internal alkenes also proved to be competent coupling partners, regardless of the $E / Z$ configuration or the position of the $\mathrm{C}=\mathrm{C}$ bond in the alkene starting material (Table 2b). As expected, styrenes themselves smoothly undergo hydroalkynylation to produce the benzylic alkynylation product exclusively (Table 2c). Under these exceptionally mild reaction conditions, various substituents on the aryl ring $\left(\mathbf{3} \mathbf{y}-\mathbf{3} \mathbf{d}^{\prime}\right)$ as well as heteroaromatic styrenes $\left(\mathbf{3} \mathbf{e}^{\prime}\right.$, 3f') were also suitable for this reaction. 
Table 2: Nickel hydride-catalyzed migratory hydroalkynylation of alkenes with bromoalkynes. ${ }^{[a]}$

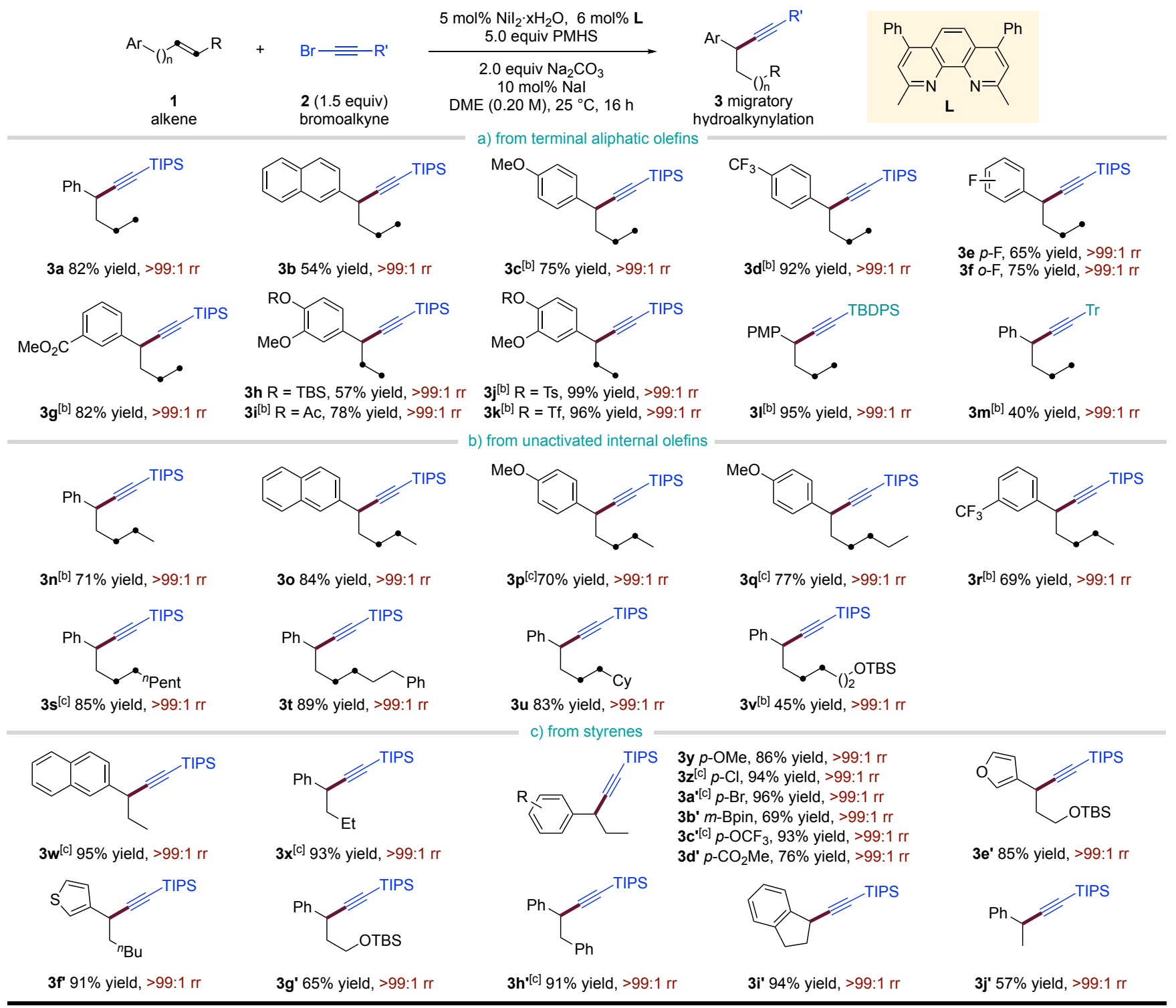

[a] Yield under each product refers to isolated yield of purified product $(0.20 \mathrm{mmol}$ scale, average of two runs); rr refers to regioisomeric ratio, representing the ratio of the major product to the sum of all other isomers as determined by GC analysis. [b] Diglyme was used as solvent. [c] DME ( $0.10 \mathrm{M})$ was used. TBDPS $=t-$ butyldiphenylsilyl; $\mathrm{Tr}=$ trityl (triphenylmethyl); TBS = $t$-butyldimethylsilyl.

In an effort to obtain enantioenriched benzylic alkynylation products, the asymmetric version of $\mathrm{NiH}$-catalyzed hydroalkynylation of styrenes was explored and the results are in Table 3. It was found that a chiral PyrOx ligand $(S)-L^{*}$ under modified reaction conditions could produce the desired hydroalkynylation products in good yields and excellent ee. Styrenes with a variety of substituents on the aromatic ring underwent asymmetric hydroalkynylation smoothly $(\mathbf{5 a}-\mathbf{5 q})$, including ethers $(\mathbf{5} \mathbf{d}-\mathbf{5 i})$, an easily reduced aldehyde $(\mathbf{5 I})$, a nitrile $(\mathbf{5 m}, \mathbf{5 n})$, and esters (5o-5q). The substituent commonly used for further cross-coupling such as aryl chloride (5c), aryl bromide (5k), and boronic acid pinacol ester (5j) all emerged unchanged. The substituents at $\beta$-position could also be varied $\left(5 \mathbf{r}-\mathbf{5} \mathbf{c}^{\prime}\right)$. Alkyl bromides were compatible with the reaction, providing a synthetic handle for further derivatization $(\mathbf{5 y}, \mathbf{5 z}) . \beta$ Unsubstituted styrenes were also compatible (5d', 5h', 5i'). The scope of bromoalkynes was also explored and a range of different sterically hindered substituents at the $\beta$-position, including silyl and alkyl substituted ethynyl bromides were shown to be viable substrates $\left(\mathbf{5} \mathbf{e}^{\prime} \mathbf{-} \mathbf{5} \mathbf{g}^{\prime}\right)$. 
Table 3: NiH-catalyzed enantioselective hydroalkynylation. ${ }^{[a, b]}$

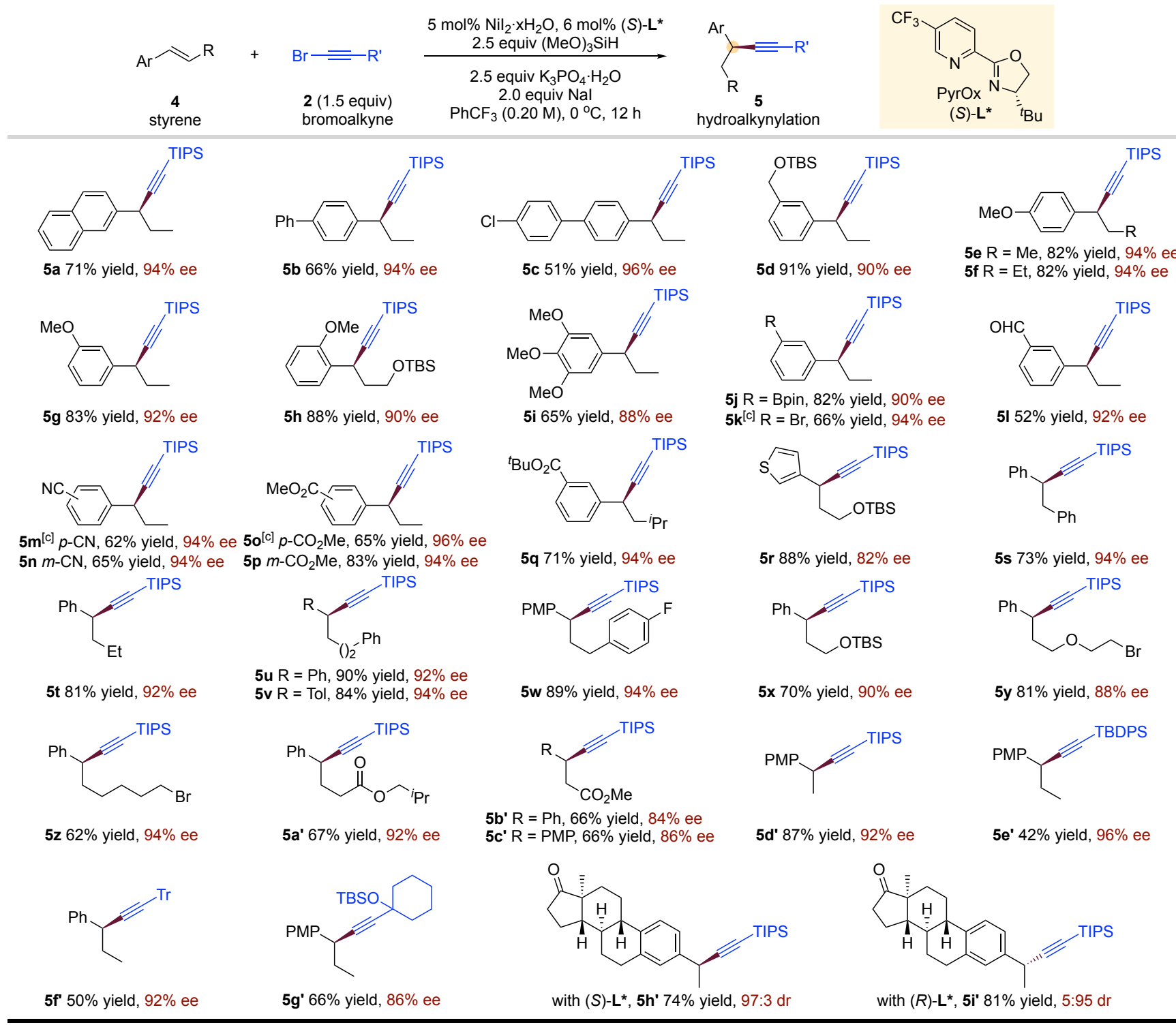

[a] Isolated yields on $0.20 \mathrm{mmol}$ scale (average of two runs). [b] Enantioselectivity was determined by chiral HPLC analysis; the absolute configuration was assigned by chemical correlation or by analogy. [c] $\mathrm{NiBr}_{2}$.diglyme used as catalyst, 1,2-dichloroethane used as solvent, 3.0 equiv $\mathrm{Nal}$ used.

When the reaction was conducted on a $5 \mathrm{mmol}$ scale, the functionalized chiral benzylic alkyne (5e) was obtained in high yield and with excellent enantioselectivity (Scheme 1a). To highlight the synthetic utility of the method, subsequent derivatizations were carried out (Scheme $1 \mathrm{~b}$ ). Desilylation of $\mathbf{5 e}$ yielded the enantioenriched terminal alkyne (6), which could further undergo a click reaction to form 7 or a hydration reaction to form 8. The semi-hydrogenation of alkyne (5a) by DIBAL-H (diisobutylaluminum hydride) could be highly stereoselective, giving the Z-alkene (9). In addition, oxidative cleavage of the triple bond in $\mathbf{5 e}$ could afford the corresponding chiral carboxylic acid (10).

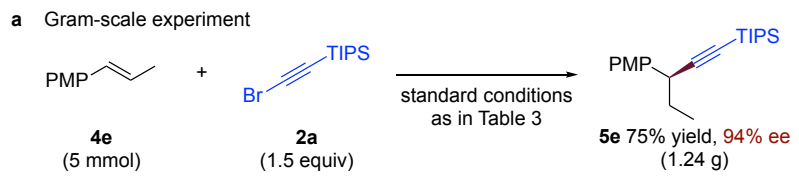

b Transformation of the enantioenriched benzylic alkynes

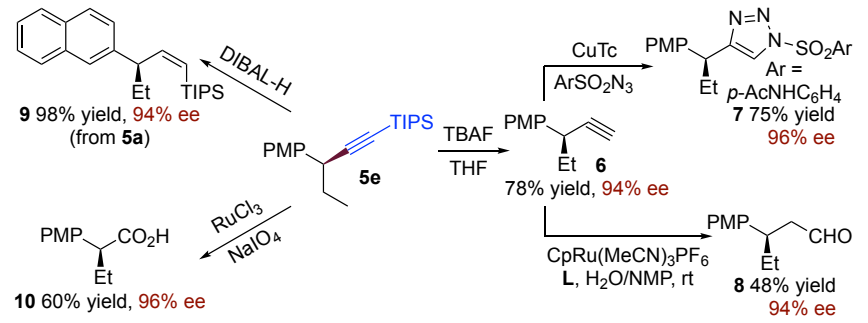

Scheme 1. Synthetic application of enantioselective hydroalkynylation. 
To gain further insight into the mechanism of the hydrometallation process, isotope labeling experiments were conducted (Scheme 2). As shown in Scheme 2a, the use of the deuterated trans-alkene (E-4h-D) led to the formation of both diastereomers in approximately equal amounts (0.55:0.45 dr), which indicated that the syn-hydrometallation is not the enantiodetermining step because if it was, a diastereomerically pure $\mathbf{5 h}$ D should be formed. This observation is consistent with our initial mechanistic proposal that the benzylic stereocenter is formed through rapid homolysis of $\mathrm{Ni}(\mathrm{III})$ and subsequently enantioconvergent process, reforming only one $\mathrm{Ni}(\mathrm{III})$ enantiomer from $\mathrm{Ni}(\mathrm{II})$ and benzylic radical (see Figure $1 \mathrm{~b}$, ii). Furthermore, no intermolecular H/D scrambled crossover products were obtained, revealing that hydrometallation of $\mathrm{NiH} / \mathrm{NiD}$ species to styrene is irreversible (Scheme $2 \mathrm{~b}$ ).

a Isotopic labelling: $\mathrm{NiH}$ syn-hydrometallation is not the enantio-determining step<smiles>[2H]/C(=C\c1ccccc1OC)C[OH2+]</smiles>

(E)-4h-D

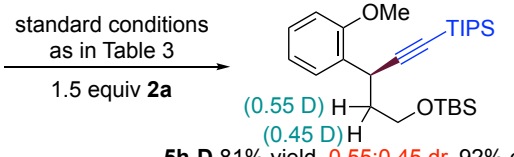

5h-D $81 \%$ yield, 0.55:0.45 dr, 92\% ee b

Crossover experiment: no intermolecular $\mathrm{H} / \mathrm{D}$ scrambled crossover products

Scheme 2. Isotopic labelling experiments.

In conclusion, we report a $\mathrm{NiH}$-catalyzed strategy to form functionalized benzylic alkynylation products, which are versatile synthetic intermediates. Both migratory hydroalkynylation and asymmetric hydroalkynylation can be realized. These two mild, efficient and straightforward processes tolerate a wide range of functional groups on both the alkene and bromoalkyne components. A broad substrate scope as well as synthetic utility of this protocol have been demonstrated. An investigation of the mechanism and the development of a migratory enantioselective version of this transformation are currently in progress.

\section{Acknowledgements}

Support was provided by NSFC $(21822105,21772087)$, NSF of Jiangsu Province (BK20190281, BK20201245), Six Kinds of Talents Project of Jiangsu Province (JNHB-003), and programs for high-level entrepreneurial and innovative talents introduction of Jiangsu Province (group program).

Keywords: alkenes • asymmetric catalysis • hydroalkynylation • nickel $\cdot$ regioselectivity
[1] For selected reviews on nickel catalysis, see: a) J. Montgomery, Organonickel Chemistry. In Organometallics in Synthesis; B. H. Lipshutz, Ed.; John Wiley \& Sons, Inc.: Hoboken, 2013; pp 319-428; b) S. Z. Tasker, E. A. Standley, T. F. Jamison, Nature 2014, 509, 299; c) G. C. Fu, ACS Cent. Sci. 2017, 3, 692; d) Nickel Catalysis in Organic Synthesis. S. Ogoshi, Ed.; Wiley-VCH: Weinheim, 2020; e) K. E. Poremba, S. E. Dibrell, S. E. Reisman, ACS Catal. 2020, 10, 8237.

[2] For selected reviews on metal-hydride chemistry, see: a) M. T. Pirnot, Y.-M. Wang, S. L. Buchwald, Angew. Chem. Int. Ed. 2016, 55, 48; b) K. D. Nguyen, B. Y. Park, T. Luong, H. Sato, V. J. Garza, M. J. Krische, Science 2016, 354, 300; c) N. A. Eberhardt, H. Guan, Chem. Rev. 2016, 116, 8373; d) S. W. M. Crossley, C. Obradors, R. M. Martinez, R. A. Shenvi, Chem. Rev. 2016, 116, 8912; e) J. Chen, J. Guo, Z. Lu, Chin. J. Chem. 2018, 36, 1075-1109; f) X.-X. Wang, X. Lu, Y. Li, J.-W. Wang, Y. Fu, Sci. China Chem. 2020, 63, 1586.

[3] For selected examples about $\mathrm{Ni}(\mathrm{I}) \mathrm{H}$, see: a) J. T. Binder, C. J. Cordier, G. C. Fu, J. Am. Chem. Soc. 2012, 134, 17003; b) J. Cornella, E. Gómez-Bengoa, R. Martin, J. Am. Chem. Soc. 2013, 135, 1997; c) C. J. Cordier, R. J. Lundgren, G. C. Fu, J. Am. Chem. Soc. 2013, 135, 10946; d) I. Pappas, S. Treacy, P. J. Chirik, ACS Catal. 2016, 6, 4105; e) Y. Kuang, D. Anthony, J. Katigbak, F. Marrucci, S. Humagain, T. Diao, Chem 2017, 3, 268.

[4] For reviews on remote functionalization via chain-walking, see: a) E. Larionov, H. Li, C. Mazet, Chem. Commun. 2014, 50, 9816; b) A. Vasseur, J. Bruffaerts, I. Marek, Nat. Chem. 2016, 8, 209; c) H. Sommer, F. Juliá-Hernández, R. Martin, I. Marek, ACS Cent. Sci. 2018, 4, 153; d) D. Janssen-Müller, B. Sahoo, S.-Z. Sun, R. Martin, Isr. J. Chem. 2020, 60, 195.

[5] For recent Zr-catalyzed chain-walking, see: a) A. Masarwa, D. Didier, T. Zabrodski, M. Schinkel, L. Ackermann, I. Marek, Nature 2014, 505, 199; b) L. Mola, M. Sidera, S. P. Fletcher, Aust. J. Chem. 2015, 68, 401; c) Y. Gao, C. Yang, S. Bai, X. Liu, Q. Wu, J. Wang, C. Jiang, X. Qi, Chem 2020, 6, 675.

[6] For recent Pd-catalyzed chain-walking, see: a) E. W. Werner, T.-S. Mei, A. J. Burckle, M. S. Sigman, Science 2012, 338, 1455; b) S. Aspin, A.-S. Goutierre, P. Larini, R. Jazzar, O. Baudoin, Angew. Chem. Int. Ed. 2012, 51, 10808; c) E. Larionov, L. Lin, L. Guénée, C. Mazet, J. Am. Chem. Soc. 2014, 136, 16882; d) T. Kochi, T. Hamasaki, Y. Aoyama, J. Kawasaki, F. Kakiuchi, J. Am. Chem. Soc. 2012, 134, 16544; e) S. Dupuy, K.-F. Zhang, A.-S. Goutierre, O. Baudoin, Angew. Chem. Int. Ed. 2016, 55, 14793; f) L. Lin, C. Romano, C. Mazet, J. Am. Chem. Soc. 2016, 138, 10344; g) Z.-M. Chen, C. S. Nervig, R. J. DeLuca, M. S. Sigman, Angew. Chem. Int. Ed. 2017, 56, 6651; h) S. Singh, J. Bruffaerts, A. Vasseur, I. Marek, Nat. Commun. 2017, 8, 14200; i) D. G. Kohler, S. N. Gockel, J. L. Kennemur, P. J. Waller, K. L. Hull, Nat. Chem. 2018, 10, 333; j) J. Bruffaerts, D. Pierrot, I. Marek, Nat. Chem. 2018, 10, 1164.

[7] For recent Co-catalyzed chain-walking, see: a) J. V. Obligacion, P. J. Chirik, J. Am. Chem. Soc. 2013, 135, 19107; b) T. Yamakawa, N. Yoshikai, Chem. Asian J. 2014, 9, 1242; c) M. L. Scheuermann, E. J. Johnson, P. J. Chirik, Org. Lett. 2015, 17, 2716; d) X. Chen, Z. Cheng, J. Guo, Z. Lu, Nat. Commun. 2018, 9, 3939.

[8] For recent $\mathrm{NiH}$-catalyzed reductive migratory hydrofunctionalization, see: a) I. Busolv, J. Becouse, S. Mazza, M. Montandon-Clerc, X. Hu, Angew. Chem. Int. Ed. 2015, 54, 14523; b) Y. He, Y. Cai, S. Zhu, J. Am. Chem. Soc. 2017, 139, 1061; c) F. Juliá-Hernández, T. Moragas, J. Cornella, R. Martin, Nature 2017, 545, 84; d) M. Gaydou, T. Moragas, F. Juliá-Hernández, R. Martin, J. Am. Chem. Soc. 2017, 139, 12161; e) F. Chen, K. Chen, Y. Zhang, Y. He, Y.-M. Wang, S. Zhu, J. Am. Chem. Soc. 2017, 139, 13929; f) F. Zhou, J. Zhu, Y. Zhang, S. Zhu, Angew. Chem. Int. Ed. 2018, 57, 4058; g) J. Xiao, Y. He, F. Ye, S. Zhu, Chem 2018, 4, 1645; h) L. Peng, Y. Li, Y. Li, W. Wang, H. Pang, G. Yin, ACS Catal. 2018, 8, 310; i) S.-Z. Sun, M. Börjesson, R. Martin-Montero, R. Martin, J. Am. Chem. Soc. 2018, 140, 12765; j) Y. Zhang, X. Xu, S. Zhu, Nat. Commun. 2019, 10, 1752; k) S.-Z. Sun, C. Romano, R. Martin, J. Am. Chem. Soc. 2019, 141, 16197; I) Y. Zhang, B. Han, S. Zhu, Angew. Chem. Int. Ed. 2019, 58, 13860; m) D. Qian, X. Hu, Angew. Chem. Int. Ed. 2019, 58, 18519; n) G. S. Kumar, A. Peshkov, A. Brzozowska, P. Nikolaienko, C. Zhu, M. Rueping, Angew. Chem. Int. Ed. 2020, 59, 
6513; p) K.-J. Jiao, D. Liu, H.-X. Ma, H. Qiu, P. Fang, T.-S. Mei, Angew. Chem. Int. Ed. 2020, 59, 6520; q) Y. Zhang, J. He, P. Song, Y. Wang, S. Zhu, CCS Chem. 2020, 2, 2259.

[9] For $\mathrm{NiH}$-catalyzed enantioselective reductive remote hydrofunctionalization, see: F. Zhou, Y. Zhang, X. Xu, S. Zhu, Angew. Chem. Int. Ed. 2019, 58, 1754.

[10] For NiH-catalyzed reductive hydrofunctionalization of olefins, see: a) $\mathrm{X}$. Lu, B. Xiao, Z. Zhang, T. Gong, W. Su, Y. Fu, L. Liu, Nat. Commun. 2016, 7, 11129; b) S. A. Green, J. L. M. Matos, A. Yagi, R. A. Shenvi, J. Am. Chem. Soc. 2016, 138, 12779; c) S. L. Shevick, C. Obradors, R. A Shenvi, J. Am. Chem. Soc. 2018, 140, 12056; d) J. Nguyen, A. Chong G. Lalic, Chem. Sci. 2019, 10, 3231; e) S. Bera, X. Hu, Angew. Chem. Int. Ed. 2019, 58, 13854.

[11] For $\mathrm{NiH}$-catalyzed enantioselective reductive hydrofunctionalization of olefins, see: a) Z. Wang, H. Yin, G. C. Fu, Nature 2018, 563, 379; b) S. J. He, J.-W. Wang, Y. Li, Z.-Y. Xu, X.-X. Wang, X. Lu, Y. Fu, J. Am. Chem. Soc. 2020, 142, 214; c) Z.-P. Yang, G. C. Fu, J. Am. Chem. Soc 2020, 142, 5870; d) S. Bera, R. Mao, X. Hu, Nat. Chem. 2021, https://doi.org/10.1038/s41557-020-00576-z; e) Y. He, C. Liu, L. Yu, S. Zhu, Angew. Chem. Int. Ed. 2020, 59, 21530; f) L. Shi, L.-L. Xing, W.-B. Hu, W. Shu, 2021, 60, 1599; g) S. Cuesta-Galisteo, J. Schörgenhumer, X. Wei, E. Merino, C. Nevado, Angew. Chem. Int. Ed. 2021, 60, 1605; h) J. Liu, H. Gong, S. Zhu, Angew. Chem. Int. Ed. https://doi.org/10.1002/anie.202012614; i) Y. He, H. Song, S. Zhu, Nat Commun. 2021, 12, 638; j) D. Qian, S. Bera, X. Hu, J. Am. Chem. Soc 2021, https://doi.org/10.1021/jacs.0c11630; k) J.-W. Wang, Y. Li, W. Nie, Z. Chang, Z.-A. Yu, Y.-F. Zhao, X. Lu, Y. Fu, ChemRxiv 2020, https://doi.org/10.26434/chemrxiv.13102307.v1; I) S. Wang, T.-Y. Zhang, J.-X. Zhang, H. Meng, B.-H. Chen, W. Shu, ChemRxiv 2020, https://doi.org/10.26434/chemrxiv.13284416.v1.

[12] For $\mathrm{NiH}$-catalyzed redox-neutral migratory hydrofunctionalization, see: a) W.-C. Lee, C.-H. Wang, Y.-H. Lin, W.-C. Shih, T.-G. Ong, Org. Lett. 2013, 15, 5358; b) J. S. Bair, Y. Schramm, A. G. Sergeev, E. Clot, O. Eisenstein, J. F. Hartwig, J. Am. Chem. Soc. 2014, 136, 13098; c) Y. He, C. Liu, L. Yu, S. Zhu, Angew. Chem. Int. Ed. 2020, 59, 9186; d) R. Yu, S. Rajasekar, X. Fang, Angew. Chem. Int. Ed. 2020, 59, 21436.

[13] For selected $\mathrm{NiH}$-catalyzed redox-neutral enantioselective hydrofunctionalization, see: a) L. Cheng, M.-M. Li, L.-J. Xiao, J.-H. Xie, Q.-L. Zhou, J. Am. Chem. Soc. 2018, 140, 11627; b) Y.-G. Chen, B. Shuai, X.-T. Xu, Y.-Q. Li, Q.-L. Yang, H. Qiu, K. Zhang, P. Fang, T.-S. Mei, J. Am. Chem. Soc. 2019, 141, 3395; c) X.-Y. Lv, C. Fan, L.-J. Xiao, J.-H. Xie, Q.-L. Zhou, CCS Chem. 2019, 1, 328; d) W.-B. Zhang, X.-T. Yang, J.-B. Ma, Z.-M. Su, S.-L. Shi, J. Am. Chem. Soc. 2019, 141, 5628; e) J. S. Marcum, T. R. Taylor, S. J. Meek, Angew. Chem. Int. Ed. 2020, 59, 14070; f) W. Shao, C. Besnard, L. Guénée, C. Mazet, J. Am. Chem. Soc. 2020, 142, 16486.

[14] For recent enantioselective syntheisis of alkyne bearing a $\alpha$-arylsubstituted sterecenter, see: a) J. Caeiro, J. P. Sestelo, L. A. Sarandeses, Chem. - Eur. J. 2008, 14, 741; b) A. Oelke, J. Sun, G. C. Fu, J. Am. Chem. Soc. 2012, 134, 2966; c) N. D. Schley, G. C. Fu, J. Am. Chem. Soc. 2014, 136, 16588; d) F.-L. Zhu, Y. Zou, D.-Y. Zhang Y.-H. Wang, X.-H. Hu, S. Chen, J. Xu, X.-P. Hu,. Angew. Chem. Int. Ed. 2014, 53, 1410; e) H. Fang, Z. Yang, L. Zhang, W. Wang, Y. Li, X. Xu, S. Zhou, Org. Lett. 2016, 18, 6022; f) X.-Y. Dong, Y.-F. Zhang, C.-L. Ma Q.-S. Gu, F.-L. Wang, Z.-L. Li, S.-P. Jiang, X.-Y. Liu, Nat. Chem. 2019, 11, 1158; g) Y. Zhang, Y. Sun, B. Chen, M. Xu, C. Li, D. Zhang, G. Zhang, Org. Lett. 2020, 22, 1490; h) L. Fu, Z. Zhang, P. Chen, Z. Lin, G. Liu, J. Am. Chem. Soc. 2020, 142, 12493; i) H.-D. Xia, Z.-L. Li, Q.-S Gu, X.-Y. Dong, J.-H. Fang, X.-Y. Du, L.-L. Wang, X.-Y. Liu, Angew. Chem. Int. Ed. 2020, 59, 16926.

[15] a) X. Zhu, J. Liu, W. Zhang, W. Nat. Chem. Biol. 2015, 11, 115; b) M. Akerman, J. Houze, D. C. H. Lin, J. Liu, J. Luo, J. C. Medina, W. Qiu, J. D. Reagan, R. Sharma, S. J. Shuttleworth, Y. Sun, J. Zhang, L. Zhu, Int. Appl. PCT, WO 2005086661, 2005. 


\section{Entry for the Table of Contents}

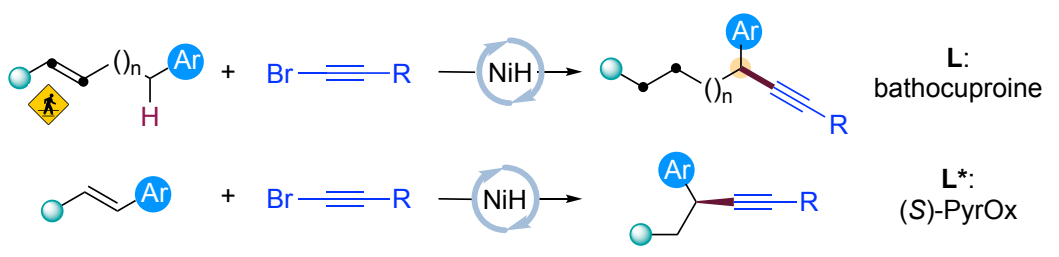

Both migratory hydroalkynylation of remote alkenes and enantioselective hydroalkynylation of styrenes processes were enabled by a reductive $\mathrm{NiH}$ strategy. A wide variety of enantioenriched benzylic alkynes, a versatile synthetic intermediate, were obtained in high yields with excellent enantioselectivities. 